\title{
Associação entre material particulado de queimadas e doenças respiratórias na região sul da Amazônia brasileira
}

\author{
Cleber Nascimento do Carmo, ${ }^{1}$ Sandra Hacon, ${ }^{1}$ Karla Maria Longo, ${ }^{2}$ \\ Saulo Freitas, ${ }^{2}$ Eliane Ignotti, ${ }^{3}$ Antonio Ponce de Leon ${ }^{4}$ e Paulo Artaxo ${ }^{5}$
}

Como citar Carmo CN, Hacon S, Longo KM, Freitas S, Ignotti E, Ponce de Leon A, et al. Associação entre material particulado de queimadas e doenças respiratórias na região sul da Amazônia brasileira. Rev Panam Salud Publica. 2010;27(1):10-6.

RESUMO Objetivo. Investigar os efeitos de curto prazo da exposição ao material particulado de queimadas da Amazônia na demanda diária de atendimento ambulatorial por doenças respiratórias de crianças e de idosos.

Métodos. Estudo epidemiológico com delineamento ecológico de séries temporais. Os registros diários de atendimento ambulatorial foram obtidos nas 14 unidades de saúde do município de Alta Floresta, Mato Grosso, região sul da Amazônia brasileira, no período de janeiro de 2004 a dezembro de 2005. Informação sobre os níveis diários de material particulado fino foi disponibilizada pelo Instituto Nacional de Pesquisas Espaciais. Para controlar possíveis fatores de confusão (situações nas quais uma associação não causal entre exposição e doença é observada devido a uma terceira variável), foram adicionadas ao modelo variáveis referentes a tendência temporal, sazonalidade, temperatura, umidade relativa do ar, precipitação pluviométrica e efeitos de calendário (como ocorrência de feriados e finais de semana). Utilizou-se regressão de Poisson via modelos aditivos generalizados.

Resultados. Um incremento de $10 \mu \mathrm{g} / \mathrm{m}^{3}$ nos níveis de exposição ao material particulado esteve associado a aumentos de 2,9 e 2,6\% nos atendimentos ambulatoriais por doenças respiratórias de crianças no $6^{\circ}$ e $7^{\circ}$ dias subsequentes à exposição. Não foram encontradas associações significativas nos atendimentos de idosos.

Conclusões. Os resultados sugerem que os níveis de material particulado das queimadas na Amazônia estão associados a efeitos adversos à saúde respiratória de crianças.

Palavras-chave Material particulado; doenças do aparelho respiratório; ambulatório hospitalar; préescolar; idoso; ecossistema amazônico; Brasil.

1 Fundação Oswaldo Cruz, Departamento de Endemias. Enviar correspondência a Cleber Nascimento do Carmo no seguinte endereço: Rua Leopoldo Bulhões 1480, sala 620, Bairro Manguinhos, CEP 21041-210, Rio de Janeiro, RJ, Brasil. E-mail: cleber@fiocruz.br, cleberdoc@yahoo.com.br.

2 Instituto Nacional de Pesquisas Espaciais (INPE), Centro de Previsão de Tempo e Estudos Climáticos (CPTEC), São Paulo (SP) Brasil.

3 Universidade do Estado de Mato Grosso (UNEMAT), Saúde Pública, Cuiabá (MT), Brasil

4 Universidade do Estado do Rio de Janeiro (UERJ), Instituto de Medicina Social, Rio de Janeiro (RJ), Brasil.

5 Universidade de São Paulo (USP), Instituto de Física, São Paulo (SP), Brasil.
As regiões do planeta que mais queimam biomassa estão concentradas nos países em desenvolvimento localizados nos trópicos e subtrópicos da África, sudeste da Ásia e América do Sul $(1,2)$. A região amazônica, por ser a maior área de floresta tropical do mundo, contendo aproximadamente um quarto de todas as florestas tropicais existentes no planeta, figura entre as regiões que possuem as maiores taxas de desmatamento (3). De acordo o Instituto Nacional de Pesquisas
Espaciais (INPE) (4), a estimativa de desmatamento na Amazônia Legal brasileira para o período de 2007 e 2008 foi de $11986 \mathrm{~km}^{2}$, um crescimento de $4 \%$ em relação ao período anterior. O desmatamento precede a fase de queimadas, com impactos diretos e indiretos sobre a saúde humana (5).

A poluição atmosférica é um preocupante problema de saúde pública em grandes centros urbanos, tendo como desfechos o aumento das internações 
hospitalares e o incremento na mortalidade (6-9). Em relação às queimadas em áreas remotas ou rurais, os poluentes gasosos e o material particulado fino apresentam efeitos diretos para o sistema respiratório, em especial para os grupos mais sensíveis $(10,11)$. Entretanto, os efeitos das queimadas sobre a saúde humana têm sido pouco estudados.

As grandes queimadas ocorridas em Bornéu (1983 e 1997), Tailândia (1997), Indonésia (1997), Estados Unidos (Califórnia, 2003) e Brasil — Roraima (1997 e 1998), Mato Grosso (1998), Pará (1998) e Acre (2005) (12) - despertaram interesse para o problema de saúde pública. As emissões de partículas finas decorrentes das queimadas representam cerca de $60 \%$ do material particulado emitido para a atmosfera (13), contribuindo de forma significativa para a alteração da composição química da atmosfera amazônica, com implicações importantes em nível local, regional e global, com valores que chegam a ultrapassar os limites observados em muitos centros urbanos (14). Estimativas revelam que a quantidade anual de material particulado liberado na atmosfera por causa de queimadas nos trópicos está em torno de 36 a $154 \mathrm{Tg}$ (15). Além dos efeitos das queimadas para o ecossistema amazônico, as emissões de poluentes contribuem para o aumento da morbidade respiratória nos municípios do chamado arco do desmatamento na região da Amazônia brasileira (11).

Diferentemente do que é observado em ambientes urbanos, em que a poluição atmosférica é caracterizada por uma exposição crônica, no caso das queimadas na Amazônia brasileira há uma exposição de elevada magnitude por um período médio anual de 3 a 5 meses, associado a baixos índices pluviométricos. Nesse período, as concentrações de material particulado menor de $10 \mu \mathrm{m}$ oriundo da queima de biomassa chegam a $400 \mu \mathrm{g} / \mathrm{m}^{3}$ (14).

Dentre os diversos poluentes liberados na queima de biomassa na Amazônia, o material particulado é o poluente que tem sido mais estudado, através do Programa de Grande Escala da BiosferaAtmosfera na Amazônia $(16,17)$. As partículas finas têm um tempo de residência na atmosfera maior do que as partículas grossas e podem ser transportadas por grandes distâncias, o que aumenta a sua capacidade de dispersão e, consequentemente, o seu impacto sobre os indiví- duos. Elas se depositam nos brônquios terminais e nos alvéolos, agravando problemas respiratórios e podendo causar mortes prematuras $(18,19)$.

Nos centros urbanos, a maioria dos agravos respiratórios detectados no atendimento ambulatorial é de menor gravidade (20) em comparação ao atendimento prestado em hospitais e serviços de emergência. Em áreas remotas da Amazônia, o atendimento prestado em ambulatórios muitas vezes substitui os hospitais e serviços de emergência. Portanto, esses atendimentos podem representar com fidelidade os efeitos da poluição do ar na saúde humana $(21,22)$. Assim, este estudo foi desenvolvido com a finalidade de analisar os efeitos da exposição ao material particulado fino $\left(\mathrm{PM}_{2.5}\right)$ oriundo das queimadas sobre os atendimentos ambulatoriais por queixas respiratórias em crianças e em idosos na região sul da Amazônia.

\section{MATERIAIS E MÉTODOS}

O Município de Alta Floresta localizase no extremo norte do Estado de Mato Grosso, a 830 km de Cuiabá, a capital do estado, no sul da Amazônia brasileira (figura 1). Possui uma população de 51136 habitantes, sendo $9 \%$ e $5 \%$ desse contingente formados por crianças e idosos, respectivamente. Apresenta um índice de desenvolvimento humano municipal (IDH-M) de 0,779, valor similar à média estadual $(0,796)$ e um pouco acima da média nacional $(0,766)$. Conta com um hospital geral, um leito hospitalar para cada 565 habitantes, 87 leitos hospitalares conveniados ao Sistema Único de Saúde (SUS) e 13 unidades básicas de saúde (UBS), 11 das quais do Programa Saúde da Família (PSF), cobrindo $64 \%$ da população. O Município de Alta Floresta vem sendo estudado desde 1992 através do Experimento de Grande Escala da Bios-

FIGURA 1. Localização do Município de Alta Floresta, Estado de Mato Grosso, Amazônia brasileira

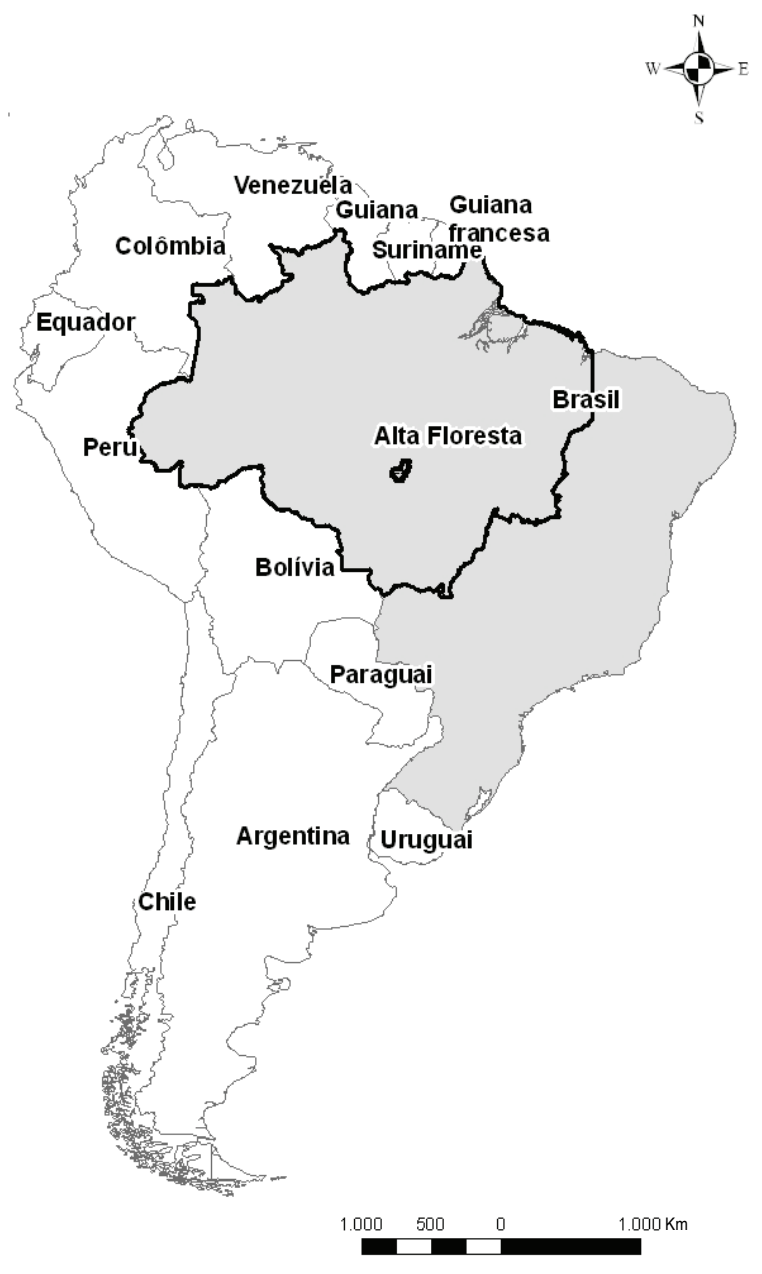


fera e Atmosfera da Amazônia (LBA) pelo Grupo de Estudos de Poluição do Ar (GEPA) do Instituto de Física da Universidade de São Paulo (IF-USP). Seu nível de desmatamento e suas queimadas vêm sendo monitorados pelo INPE.

$\mathrm{O}$ presente estudo epidemiológico, com delineamento ecológico, foi conduzido por meio de séries temporais com registros diários de atendimento ambulatorial por doenças respiratórias de crianças menores de 5 anos de idade e de idosos maiores de 64 anos nas 14 unidades de saúde do município de Alta Floresta, no período de $1^{\circ}$ de janeiro de 2004 a 31 de dezembro de 2005. Também foram utilizadas medidas de concentrações diárias de material particulado fino e variáveis meteorológicas e de calendário (como dias da semana e feriados). Os grupos etários foram selecionados por serem os mais vulneráveis aos efeitos da poluição atmosférica (19). Foram excluídos os registros de atendimentos de urgência/ emergência em razão da ausência de dados sistematizados de consultas realizadas no hospital municipal, onde se localiza o pronto-socorro do Município.

A classificação dos registros de atendimentos ambulatoriais por doenças respiratórias foi feita de acordo com a Segunda Versão da Classificação Internacional de Atenção Primária (ICPC-2), e não como preconiza a Décima Revisão da Classificação Estatística Internacional de Doenças e Problemas Relacionados à Saúde (CID-10), em razão das limitações desta última para classificar atendimentos na esfera da atenção primária (23, 24). Medidas diárias de $\mathrm{PM}_{2.5}$ foram estimadas por modelos matemáticos desenvolvidos pelo INPE. Entre eles, um modelo numérico desenvolvido pelo INPE (Coupled Aerosol and Tracer Transport model to the Brazilian developments on the Regional Atmospheric Modeling System, CATT-BRAMS) estima a concentração de poluentes atmosféricos derivados da queima de biomassa na região amazônica (25). O CATT-BRAMS fornece medidas de $\mathrm{PM}_{2.5}$ para cada período de 3 horas. A partir desses dados, foram calculadas médias aritméticas diárias da concentração de material particulado fino. Informações meteorológicas diárias (temperatura, umidade relativa do ar e total de precipitação) foram obtidas do Centro de Previsão do Tempo (CPTEC)/INPE.

Considerando que a magnitude das queimadas aumenta no período de bai- xos índices pluviométricos e diminui a praticamente zero nos períodos de chuva, foram realizadas análises independentes para os dois períodos. Com base nos níveis diários de material particulado e totais pluviométricos, optou-se por definir o período mais crítico para a prática de queimadas como sendo entre os meses de abril a novembro.

Foram calculadas estatísticas descritivas para o $\mathrm{PM}_{2.5}$, média diária de temperatura, média diária da umidade relativa do ar, precipitação pluviométrica e atendimentos ambulatoriais por doenças respiratórias em crianças e em idosos. Para verificar a relação existente entre o poluente, os fatores meteorológicos e a variável de desfecho foram calculados coeficientes de correlação linear de Pearson.

O número diário de atendimentos ambulatoriais por doenças respiratórias foi considerado como variável dependente e os níveis médios diários de material particulado fino foram analisados como variável independente. As variáveis de controle introduzidas nos modelos foram: dia da semana, feriados, número de dias transcorridos (desde o início do período), média diária de temperatura, média diária da umidade relativa do ar e precipitação pluviométrica, estas três últimas para controle de efeito meteorológico.

Para o tratamento estatístico, as contagens diárias dos atendimentos de crianças e de idosos foram modeladas separadamente em regressões de Poisson. Para estimar a associação existente entre as variações diárias nas concentrações de material particulado fino e os totais diários de atendimento ambulatorial por doenças respiratórias, foram utilizados modelos aditivos generalizados (26). Esses modelos permitem que efeitos não lineares sejam ajustados de forma adequada utilizando-se funções não paramétricas. No presente caso, fez-se uso de funções splines, que suavizam as curvas de análise. Assumiu-se uma relação linear entre os atendimentos ambulatoriais e o material particulado fino. Para cada grupo etário, estimou-se o impacto dos níveis de material particulado na demanda por atendimento ambulatorial em dois períodos distintos: período de queimadas (abril a novembro) e período sem queimadas (dezembro a março).

As respostas biológicas da saúde humana aos efeitos adversos da poluição atmosférica apresentam aparentemente um comportamento defasado em relação ao período de exposição do indivíduo ao poluente atmosférico $(7,8,27,28)$. Isso significa que os atendimentos realizados em um dado dia podem estar relacionados tanto à poluição do referido dia como também à poluição de dias anteriores. Nesse sentido, optou-se por utilizar modelos com defasagens de até 7 dias após a exposição ao poluente $(7,28)$.

Os efeitos estimados são riscos relativos (RR) correspondentes a aumentos de $10 \mu \mathrm{g} / \mathrm{m}^{3}$ na exposição ao $\mathrm{PM}_{2.5}$. Para uma melhor interpretação dos resultados, os RR foram convertidos para aumentos percentuais. Todas as análises foram realizadas por meio do programa estatístico $R$ versão 2.7 (29) e da biblioteca ares (30), uma coleção de rotinas para análise de dados de séries temporais no programa estatístico R. Os níveis de significância adotados foram de 5 e $10 \%$.

\section{RESULTADOS}

Durante os anos de 2004 e 2005, foram realizados 1646 atendimentos de crianças e 262 de idosos por todas as causas respiratórias nas 14 unidades de saúde do Município de Alta Floresta. A tabela 1 mostra as estatísticas descritivas das variáveis no período do estudo. A média diária de atendimento ambulatorial por doenças respiratórias para as crianças foi 5,8 vezes maior do que para os idosos. A média aritmética diária de $\mathrm{PM}_{2.5}$ representa um indicador de exposição diária à poluição atmosférica por queima de biomassa no Município de Alta Floresta. Verificou-se que, durante o período de prática de queimadas, a concentração média diária de $\mathrm{PM}_{2.5}$ ultrapassou os níveis de exposição de $25 \mu \mathrm{g} / \mathrm{m}^{3}$, considerados aceitáveis pela Organização Mundial da Saúde (OMS) (31).

A análise de correlação no período de queimadas (tabela 2) indica que, além de existir uma associação linear direta entre o poluente e o desfecho, há uma correlação entre o poluente e as variáveis meteorológicas. As séries temporais dos atendimentos ambulatoriais não apresentam um comportamento sazonal consistente com a série diária de material particulado. Contudo, o $\mathrm{PM}_{2.5}$ esteve significativamente associado com maior número de atendimentos ambulatoriais por doenças respiratórias de crianças. Variações diárias de concentração de $\mathrm{PM}_{2.5}$ estiveram estatisticamente associadas com o aumento na demanda diária de atendimento ambulatorial de crianças, mesmo após controle de efeitos de confusão, isto 
TABELA 1. Estatísticas descritivas dos atendimentos ambulatoriais por doenças respiratórias em crianças e idosos, variáveis meteorológicas e dados de $\mathrm{PM}_{2.5}$, Alta Floresta (MT), Brasil, 2004 e $2005^{a}$

\begin{tabular}{lcccr}
\hline & Média diária & Desvio-padrão & Mínimo & Máximo \\
\hline Atendimentos & & & & \\
$\quad$ Crianças & 2,3 & 2,7 & 0 & 18 \\
$\quad$ Idosos & 0,4 & 0,7 & 0 & 4 \\
Variáveis meteorológicas & & & & \\
$\quad$ Temperatura $\left({ }^{\circ} \mathrm{C}\right)$ & 26,8 & 4,4 & 0 & 36,3 \\
Umidade relativa $(\%)$ & 68,0 & 16,7 & 0 & 98,1 \\
$\quad$ Precipitação $(\mathrm{mm})$ & 7,1 & 15,9 & 0 & 113,0 \\
$\mathrm{PM}_{2.5}$ & & 33,2 & 0,1 & 257,4 \\
Completo $(731$ dias) & 22,8 & 36,6 & 0,1 & 257,4 \\
Queimadas & 32,9 & 3 & 0,1 & 15,9 \\
$\quad$ Sem queimadas & 2,5 & & &
\end{tabular}

${ }^{a}$ Durante 731 dias.

TABELA 2. Matriz de correlação de Pearson das variáveis sob estudo no período de queimadas (abril a novembro), Alta Floresta (MT), Brasil, 2004 a 2005

\begin{tabular}{lcccccc}
\hline & $\mathrm{PM}_{2.5}$ & Temperatura & Umidade & Precipitação & Crianças & Idosos \\
\hline $\mathrm{PM}_{2.5}$ & 1,00 & & & & & \\
Temperatura & $0,21^{\mathrm{a}}$ & 1,00 & & & & \\
Umidade & $-0,38^{\mathrm{a}}$ & $-0,52^{\mathrm{a}}$ & 1,00 & & & \\
Precipitação & $-0,20^{\mathrm{a}}$ & $-0,33^{\mathrm{a}}$ & $0,38^{\mathrm{a}}$ & 1,00 & & \\
Crianças & $0,14^{\mathrm{a}}$ & $0,08^{\mathrm{a}}$ & $-0,05$ & $-0,11^{\mathrm{a}}$ & 1,00 & \\
Idosos & 0,02 & 0,06 & $-0,01$ & $-0,07$ & $0,34^{\mathrm{a}}$ & 1,00 \\
\hline
\end{tabular}

a $P<0,05$.

é, quando o modelo foi controlado por variáveis que apresentavam correlação tanto com o desfecho quanto com a exposição. Foram encontradas associações significativas aos níveis de 5 e $10 \%$, no período de prática de queimadas, no $6^{\circ}$ e $7^{\circ}$ dias após a exposição ao poluente, com aumentos de 2.9\% (IC95\%: 0,3 a 5,$5 ; P=0,031$ ) e $2.6 \%$ (IC95\%: 0,0 a 5,4 ; $P=0,063)$ no número de atendimentos ambulatoriais, respectivamente.

Nenhuma associação foi evidenciada para o atendimento ambulatorial de idosos. A representação gráfica das associações, em forma de aumentos percentuais estimados, entre os atendimentos ambulatoriais por doenças respiratórias devido a aumentos de $10 \mu \mathrm{g} / \mathrm{m}^{3}$ na concentração de $\mathrm{PM}_{2.5}$ e os respectivos intervalos de confiança são exibidos nas figuras 2 e 3 .

\section{DISCUSSÃO}

Este estudo evidenciou que a exposição ao material particulado fino oriundo da fumaça das queimadas está associada ao aumento da demanda por atendimento ambulatorial por doenças respiratórias em crianças residentes no município de Alta Floresta, região do arco do desmatamento na Amazônia brasileira. Os resultados encontrados corroboram outros achados descritos na literatura nacional $(11,32,33)$ e internacional $(34,35)$. Apesar dos pequenos valores percentuais de incremento no número diário de atendimentos ambulatoriais, o impacto da exposição ao $\mathrm{PM}_{2.5}$ é substancial quando se considera o contingente populacional sob exposição.

Botelho et al. (36) verificaram uma probabilidade 7,3\% maior de internação hospitalar no período de seca se comparado ao das chuvas ao analisar atendimentos de urgência por doenças respiratórias em Cuiabá, cidade também localizada na Amazônia brasileira. Sabe-se que parte dos casos que demandam consulta médica na rede básica demandarão também internações hospitalares ou poderão chegar a óbito. Sendo assim, é provável que o incremento percentual no risco de aumento na demanda diária por atendimentos ambulatoriais seja menor se comparado ao risco verificado na literatura em termos de admissão hospitalar (33, 37); entretanto, em termos absolutos, a demanda por atendimento ambulatorial envolve maior número de indivíduos.

O atendimento ambulatorial por doenças respiratórias, embora possa ser considerado de menor gravidade quando comparado às internações e aos atendimentos de emergência, é de grande importância para a saúde pública na Amazônia. Devido à existência de muitos povoados distantes dos centros urbanos, os pacientes não têm como chegar aos hospitais e serviços de emergência por várias razões, como falta de transporte público, problemas econômicos e número limitado de hospitais para atendimento da população (5).

Também é necessário considerar que o atendimento ambulatorial tem elevada frequência em áreas remotas ou rurais da Amazônia, indicando a magnitude dos impactos negativos das doenças respiratórias na qualidade de vida das pessoas e de consequências econômicas como o absenteísmo na escola e no trabalho (27). Essa característica da exposição na Amazônia pode explicar parcialmente a diferença na magnitude do efeito ao $\mathrm{PM}_{2.5}$, mais prolongado, com impacto 6 a 7 dias após a exposição, se caracterizando mais como um efeito crônico. Uma hipótese é que os pacientes aguardam até uma situação mais grave para procurar os serviços de saúde.

As estimativas de $\mathrm{PM}_{2.5}$ são de grande interesse para a saúde pública, principalmente na Amazônia, já que são um indicador de exposição à fumaça das queimadas. $\mathrm{O}$ uso de estimativas de $\mathrm{PM}_{25}$ geradas pelo modelo CATT-BRAMS foi crucial para este estudo. A implantação de uma rede automática de monitoramento de poluição do ar na Amazônia é inviável, seja pelo elevado custo de manutenção, seja pela falta de infraestrutura física e de pessoal treinado para operá-la. O uso dos dados gerados pelo modelo CATT-BRAMS enfatiza a necessidade de o INPE disponibilizar dados diários de material particulado oriundo das queimadas para que outros grupos possam realizar estudos semelhantes e aumentar a discussão sobre o tema.

Em contraste com a grande quantidade de informações relativas à poluição atmosférica em áreas urbanas, há escassez de estudos relacionados à poluição por queimadas. Os estudos da Amazônia poderiam ser comparados com os estudos oriundos da queima da cana- 
FIGURA 2. Incremento percentual e intervalos de confiança para atendimentos ambulatoriais por doenças respiratórias de crianças em função do aumento de $10 \mu \mathrm{g} / \mathrm{m}^{3}$ na concentração do $\mathrm{PM}_{2.5}$ durante períodos com e sem queima de biomassa, Alta Floresta (MT), Brasil, 2004 e 2005

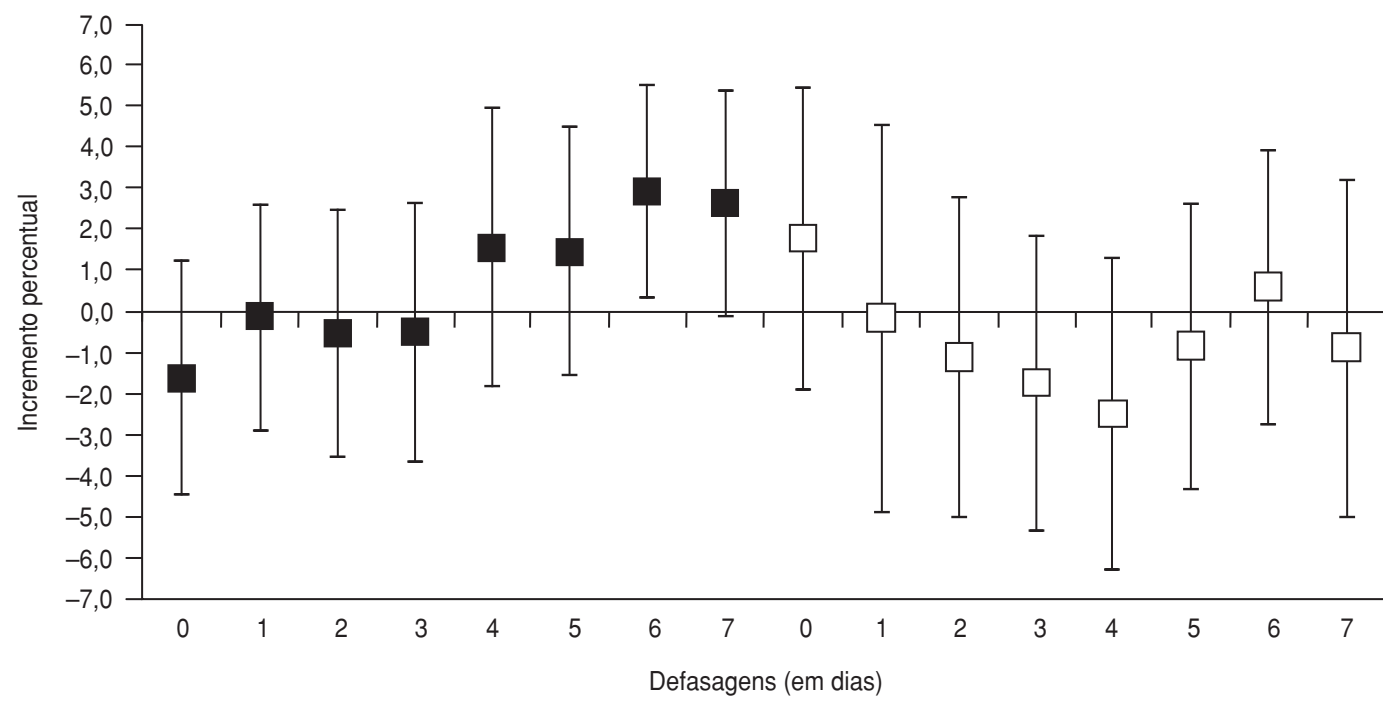

Período de queima de biomassa

Período sem queima de biomassa

FIGURA 3. Incremento percentual e intervalos de confiança para atendimentos ambulatoriais por doenças respiratórias de idosos em função do aumento de $10 \mu \mathrm{g} / \mathrm{m}^{3}$ na concentração do $\mathrm{PM}_{2.5}$ durante períodos com e sem queima de biomassa, Alta Floresta (MT), Brasil, 2004 e 2005

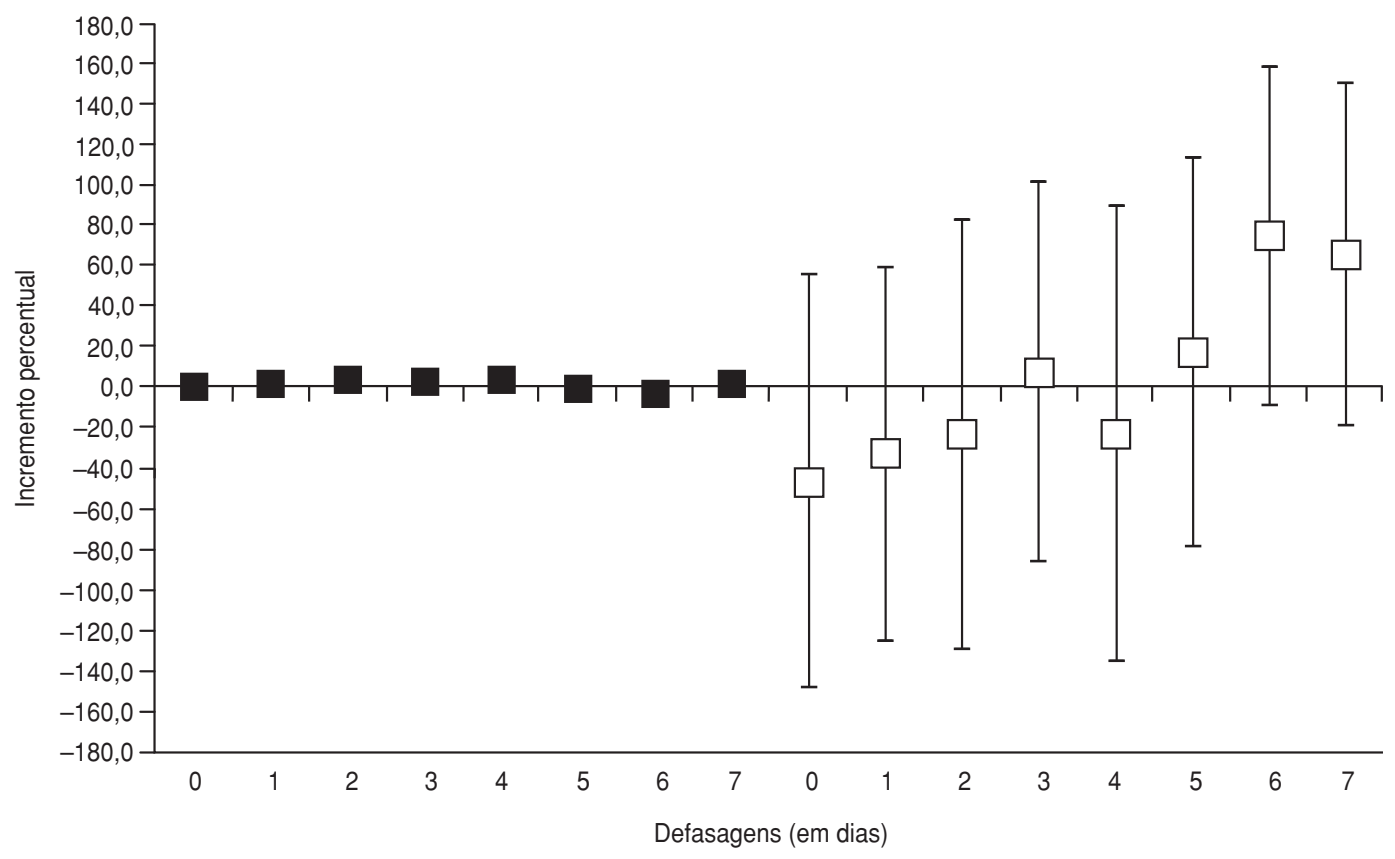

Período de queima de biomassa

Período sem queima de biomassa

de-açúcar, realizados, principalmente, na Região Sudeste do país $(32,33)$. Entretanto, as características de exposição humana são diferentes, porque nas regiões de queima de cana-de-açúcar também há indústrias, além da grande quantidade de veículos, o que implica em composição química diferenciada das condições de exposição da Amazônia. Outra importante diferença entre a Amazônia e outras áreas é que na região amazônica, durante a estação chuvosa, não há po- luição do ar, com exceção de algum particulado biogênico na atmosfera (17).

Embora o tamanho das partículas provenientes da queima de biomassa seja conhecido como determinante para a magnitude do agravo à saúde, no Brasil a 
legislação vigente (38) não faz distinção quanto ao tamanho da partícula, assim como não contempla as características da exposição aguda da Amazônia. A OMS estabeleceu como limite anual de exposição para o $\mathrm{PM}_{2.5}$ a média de $10 \mu \mathrm{g} / \mathrm{m}^{3}$, com uma média diária de $25 \mu \mathrm{g} / \mathrm{m}^{3}$ para áreas urbanas (31), sem distinguir o tipo de emissão e o tipo de exposição (aguda ou crônica). Apesar de o período estudado (2004 e 2005) ter sido considerado de baixa exposição, com $70 \%$ das medidas de $\mathrm{PM}_{2.5}$ abaixo de $25 \mu \mathrm{g} / \mathrm{m}^{3}$, o limite definido pela OMS poderia ser usado pelas secretarias de saúde da região Amazônica como um limite de proteção à saúde humana. O mecanismo fisiológico pulmonar, combinado com o elevado nível de exposição, poderia explicar por que em alguns municípios da região amazônica ocorre alta prevalência de doenças respiratórias nos grupos etários mais vulneráveis, como crianças e idosos (10).

Salienta-se como limitação da qualidade dos registros de atendimentos ambulatoriais o fato de esses não possuírem padronização e validação de diagnósticos. Tais limitações impossibilitaram uma análise mais detalhada do grupo das doenças respiratórias segundo diagnósticos ou vias aéreas atingidas. Porém, ainda que o estudo refira-se a dados secundários, a ausência de vinculação do diagnóstico ao faturamento financeiro no nível da atenção básica torna mais relevantes os achados deste estudo. Mesmo que haja alguma discrepância em relação ao diagnóstico, é pouco provável que se refira a outro agravo não decorrente do sistema respiratório (24).

Ao analisar os registros de atendimentos da rede pública, os dados refletem os atendimentos realizados pela parcela da população que utiliza esse serviço, que corresponde à maior parte da população da região. Adicionalmente, nos estudos ecológicos diários de séries temporais, fatores cuja distribuição não varia diariamente não representam possíveis variáveis de confusão. A qualidade das informações de saúde e demais problemas dessa natureza na base de dados não va- riam diariamente e não são correlacionados com o poluente. Logo, suas consequências sobre as estimativas de efeitos encontradas seriam mínimas.

Espera-se que os resultados aqui apresentados possam contribuir para um melhor entendimento do impacto da poluição atmosférica por queima de biomassa na saúde das pessoas, em particular crianças e idosos, residentes na Amazônia brasileira.

Agradecimentos. Este estudo é parte do projeto intitulado "Avaliação dos efeitos da queima de biomassa na saúde humana na Amazônia brasileira", inserido no projeto "Integração de abordagens do ambiente, uso da terra e dinâmica social na Amazônia: as relações homem-ambiente e o desafio da sustentabilidade - LBA2 Milênio", financiado com recursos do Conselho Nacional de Desenvolvimento Científico e Tecnológico (CNPq), Fundação Oswaldo Cruz - PAPES IV e do Fundo de Apoio à Pesquisa de Mato Grosso (FAPEMAT).

\section{REFERÊNCIAS}

1. Chand D, Guyon P, Artaxo P, Schmid O, Frank GP, Rizzo LV, et al. Optical and physical properties of aerosols in the boundary layer and free troposphere over the Amazon Basin during the biomass burning season. Atmos Chem Phys. 2006;6(10):2911-25.

2. Ichoku C, Giglio L, Wooster MJ, Remer LA. Global characterization of biomass-burning patterns using satellite measurements of fire radioactive energy. Remote Sens Environ. 2008;112(6):2950-62.

3. Guyon P, Frank GP, Welling M, Chand D, Artaxo P, Rizzo L, et al. Airborne measurements of trace gas and aerosol particle emissions from biomass burning in Amazonia. Atmos Chem Phys. 2005;5(11):2989-3002.

4. Brasil, Ministério da Ciência e Tecnologia, Instituto Nacional de Pesquisas Espaciais. Disponível em: www.obt.inpe.br/prodes/index.html. Acessado em 12 de dezembro de 2008.

5. Confalonieri UEC. Saúde na Amazônia: um modelo conceitual para a análise de paisagens e doenças. Estud Av. 2005;19(53):221-36.

6. Saldiva PHN. Efeitos da poluição atmosférica na morbidade e mortalidade em São Paulo. Braz J Med Biol Res. 1996;29(9):1195-9.

7. Freitas C, Bremner SA, Gouveia N, Pereira LAA, Saldiva PHN. Internações e óbitos e sua relação com a poluição atmosférica em São Paulo, 1993 a 1997. Rev Saude Publica. 2004; 38(6):751-7.

8. Hong YC, Lee JT, Kim H, Ha EH, Schwartz J, Christiani DC. Effects of air pollutants on acute stroke mortality. Environ Health Perspect. 2002;110:187-91.
9. Pope CA 3rd, Burnett RT, Thun MJ, Calle EE, Krewski D, Ito K, et al. Lung cancer, cardiopulmonary mortality, and long-term exposure to fine particulate air pollution. JAMA. 2002;287(9):1132-41.

10. Ignotti E, Hacon SS, Silva AMC, Junger WL, Castro $\mathrm{H}$. Efeitos das queimadas na Amazônia: método de seleção dos municípios segundo indicadores de saúde. Rev Bras Epidemiol. 2007;10(4):453-64.

11. Mascarenhas MDM, Vieira LC, Lanzieri TM, Leal APPR, Duarte AF, Hatch DL. Poluição atmosférica devida a queima de biomassa florestal e atendimentos de emergência por doença respiratória em Rio Branco, Brasil - setembro 2005. J Bras Pneumol. 2008;34(1):42-6.

12. Cochrane ME. O grande incêndio de Roraima. Cienc Hoje. 2000;27(157):26-43.

13. Hacon S, Artaxo P, Gerab F, Yamasoe MA, Campos RC, Conti LF, et al. Atmospheric mercury and trace elements in the region of Alta Floresta in the Amazon basin. Water Air Soil Pollut. 1995;80(1-4):273-83.

14. Artaxo P, Martins JV, Yamasoe MA, Procópio AS, Pauliquevis TM, Andreae MO, et al. Physical and chemical properties of aerosols in the wet and dry seasons in Rondônia, Amazonia. J Geophys Res. 2002;107(D20):8081.

15. Crutzen PJ, Andreae MO. Biomass burning in the tropics: impact on atmospheric chemistry and biogeochemical cycles. Science. 1990; 250(4988):1669-78.

16. Brasil, Ministério da Ciência e Tecnologia. Programa de Grande Escala da Biosfera - Atmosfera na Amazônia. Disponível em: lba.cptec. inpe.br/lba/site/. Acessado em 3 de fevereiro de 2009.

17. Artaxo P, Oliveira PH, Lara LL, Pauliquevis TM, Rizzo LV, Junior CP, et al. Efeitos climáticos de partículas de aerossóis biogênicos e emitidos em queimadas na Amazônia. Rev Bras Meteorol. 2006;21(3):168-22.

18. Ibaldi-Mulli A, Wichmann HE, Kreyling W, Peters A. Epidemiological evidence on health effects of ultrafine particles. J Aerosol Med. 2002;15(2):189-201.

19. Pope CA 3rd. Epidemiology of fine particulate air pollution and human health: biologic mechanisms and who's at risk? Environ Health Perspect. 2000;108 Suppl 4:713-23.

20. Wong TW, Tam W, Tac Sun Yu I, Wun YT, Wong $\mathrm{AH}$, Wong CM. Association between air pollution and general practitioner visits for respiratory diseases in Hong Kong. Thorax. 2006;61(7):585-91.

21. $\mathrm{Xu} X, \mathrm{Li} B$, Huang $\mathrm{H}$. Air pollution and unscheduled hospital outpatient and emergency room visits. Environ Health Perspect. 1995; 103(3):286-9.

22. Hajat $S$, Anderson HR, Atkinson RW, Haines A. Effects of air pollution on general practitioner consultations for upper respiratory diseases in London. Occup Environ Med. 2002; 59(5):294-9.

23. International Classification of Primary Care, ICPC-2. $2^{\mathrm{a}}$ ed. Oxford: Oxford University Press; 1999.

24. Rosa AM, Ignotti E, Botelho C, Castro HA, Hacon SS. Doença respiratória e sazonalidade climática em menores de 15 anos em um mu- 
nicípio da Amazônia brasileira. J Pediatr (Rio J). 2008;84(6):543-9.

25. Longo KM, Freitas SR, Setzer A, Prins E, Artaxo P, Andreae M. The Coupled Aerosol and Tracer Transport model to the Brazilian developments on the Regional Atmospheric Modeling System (CATT-BRAMS). Part 2: Model sensitivity to the biomass burning inventories. Atmos Chem Phys. 2007;7:8571-95.

26. Hastie T, Tibshirani R. Generalized additive models. Londres: Chapman \& Hall; 1990.

27. Gouveia N, Freitas CU, Martins LC, Marcilio IO. Hospitalizações por causas respiratórias e cardiovasculares associadas à contaminação atmosférica no Município de São Paulo, Brasil. Cad Saude Publica. 2006;22(12):2669-77.

28. Lee SL, Wong WHS, Lau YL. Association between air pollution and asthma admission among children in Hong Kong. Clin Exp Allergy. 2006;36(9):1138-46.

29. R Development Core Team. R: A language and environment for statistical computing. Viena: R Foundation for Statistical Computing; 2008.
30. Junger WL. Análise, imputação de dados e interfaces computacionais em estudos de séries temporais epidemiológicas [tese]. Rio de Janeiro: Universidade do Estado do Rio de Janeiro; 2008.

31. World Health Organization. Air quality guidelines: global update. Genebra: WHO. 2005.

32. Cançado JE, Saldiva PH, Pereira LA, Lara LB, Artaxo P, Martinelli LA, et al. The impact of sugar cane-burning emissions on the respiratory system of children and the elderly. Environ Health Perspect. 2006;114(5):725-9.

33. Arbex MA, Martins LC, de Oliveira RC, Pereira LA, Arbex FF, Cançado JE, et al. Air pollution from biomass burning and asthma hospital admissions in a sugar cane plantation area in Brazil. J Epidemiol Community Health. 2007;61(5):395-400.

34. Hisham-Hashim J, Hashim Z, Jalaludin J, Lubis SH, Hashim R. Respiratory function of elementary school children exposed to the 1997 Kuala Lumpur haze. Epidemiology. 1998;9(4):S1.
35. Lipsett M, Hurley S, Ostro B. Air pollution and emergency room visits for asthma in Santa Clara County, California. Environ Health Perspect. 1997;105(2):216-22

36. Botelho C, Correia AL, Silva AMC, Macedo AG, Clystenes OS. Fatores ambientais e hospitalizações em crianças menores de cinco anos com infecção respiratória aguda. Cad Saude Publica. 2003;19(6):1771-80.

37. Arbex MA, Cançado JED, Pereira LAA, Braga ALF, Saldiva PHN. Queima de biomassa e efeitos sobre a saúde. J Bras Pneumol. 2004; 30(2):158-75.

38. Brasil, Conselho Nacional de Meio Ambiente (CONAMA). Resolução N. ${ }^{\circ} 003$ de 28 de junho de 1990. Brasília: Diário Oficial da União. Disponível em: www.mma.gov.br/ port/conama/res/res90/res0390.html. Acessado em 25 de janeiro de 2009.

Manuscrito recebido em 4 de maio de 2009. Aceito em versão revisada em 3 de junho de 2009.

ABSTRACT Objective. To investigate the short-term effects of exposure to particulate matter from biomass burning in the Amazon on the daily demand for outpatient care due to respiratory diseases in children and the elderly.

Association between particulate matter from biomass burning and respiratory diseases in the southern region of the Brazilian Amazon

Key words
Methods. Epidemiologic study with ecologic time series design. Daily consultation records were obtained from the 14 primary health care clinics in the municipality of Alta Floresta, state of Mato Grosso, in the southern region of the Brazilian Amazon, between January 2004 and December 2005. Information on the daily levels of fine particulate matter was made available by the Brazilian National Institute for Spatial Research. To control for confounding factors (situations in which a non-causal association between exposure and disease is observed due to a third variable), variables related to time trends, seasonality, temperature, relative humidity, rainfall, and calendar effects (such as occurrence of holidays and weekends) were included in the model. Poisson regression with generalized additive models was used.

Results. A $10 \mu \mathrm{g} / \mathrm{m}^{3}$ increase in the level of exposure to particulate matter was associated with increases of $2.9 \%$ and $2.6 \%$ in outpatient consultations due to respiratory diseases in children on the 6th and 7th days following exposure. Significant associations were not observed for elderly individuals.

Conclusions. The results suggest that the levels of particulate matter from biomass burning in the Amazon are associated with adverse effects on the respiratory health of children.

Particulate matter; respiratory tract diseases; outpatient clinics, hospital; child, preschool; aged; Amazonian ecosystem; Brazil. 